\title{
Subject Index Volume 11, 1998
}

Key to Abbreviations

bkrv - book review
bn - board news
br - brief report
c - correspondence
crHIV - current report - HIV
cr - clinical review
e- editorial
ebcp - evidence-based clinical practice
g - guidelines

Abdomen, Acute. An unusúal cause of ureter rupture and acute abdomen (Fink) 318-br

Abdominal Pain. Osteitis pubis: a diagnosis for the family physician (Andrews \& Carek) 291-mp

Acetaminophen/adverse effects. Acute renal failure in an alcoholic patient taking therapeutic doses of acetaminophen (Maze \& Lee) 410-br

Acidosis, Lactic. Metformin-associated lactic acidosis (Hulisz et al) 233-br

Acquired Immunodeficiency Syndrome. Oral health care issues in HIV disease: developing a core curriculum for primary care physicians (Sifri et al) 434-oa.

Put to sleep (Magill) 165-refl

Activities of Daily Living. Clinical feasibility of a free-weight strength-training program for seniors (Brill et al) Nov-oa

Acupuncture Therapy. The use of alternative health care by a family practice population (Drivdahl \& Miser) 193-oa; Alternative medicine and the family physician (Jonas) 244-e

Adrenal Cortex Hormones. The use of corticosteroids in the management of herpes zoster (MacFarlane et al) 224-step

Adrenaline - see Epinephrine.

Aged. A gathering of eagles (Saal) 240-refl

Approaches to urinary incontinence in a rural population: a comparison of physician assistants, nurse practitioners, and family physicians (Jones \& Bunner) 207-mp

Clinical feasibility of a free-weight strength-training program for seniors (Brill et al) 445-oa

Gastrostomies in older patients: the 1990 National Hospital Discharge Survey (Grant) 187-oa; A gastrostomy in every stomach? (Brummel-Smith) 242-e

Medical consultation for the elderly patient with hip fracture (Ackermann) 366-cr

Recognition and management of oral health problems in older adults by physicians: a pilot study Jones et al) 474-br

AIDS virus - see HIV.

Alcoholism. Acute renal failure in an alcoholic patient taking therapeutic doses of acetaminophen (Maze \& Lee) 410-br
hos - health care system
$\mathrm{hp}$ - health policy
$\mathrm{mp}$ - medical practice
oa - original article
refl - reflections in family practice
$\mathrm{sp}$ - special communication
step - STEPped care
$\mathrm{w}$ - world perspective
c-correction

alpha-Fetoproteins. Influence of provider characteristics and insurance status on maternal serum alphafetoprotein screening Jenkins-Woelk et al) 357-oa

Alternative Medicine. The use of alternative health care by a family practice population (Drivdahl \& Miser) 193-oa; Alternative medicine and the family physician (Jonas) 244-e

Alternative medicine/education. Primary care physicians and complementary-alternative medicine: training, attitudes, and practice patterns (Berman et al) 272-oa

Alzheimer's Disease. Evaluating driving performance of outpatients with Alzheimer disease (Cox et al) 264-oa

Amantadine. Management of Parkinson disease (Mandanas) 171-c; (Manyam) 171-c

American Board of Family Practice. A conversation with the ABFP executive director (Avant) 420-bn

Pisacano Memorial Foundation - continuing the tradition (Cattoi) 490-bn

Ammonia. Acute-onset persistent olfactory deficit resulting from multiple overexposures to ammonia vapor at work (Prudhomme et al) 66-br; Irritant-induced anosmia (Edwards) 497-c; (Blanc) 497-c

Anesthesia, Local. Advances in office anesthesia (Whealton) 200-cr

Anti-Anxiety Agents, Benzodiazepine. Pharmacotherapy of panic disorder: proposed guidelines for the family physician (Roy-Byrne et al) 282-cr

Anticholesteremic Agents. Evidence-based medicine in primary care: an overview (Geyman) 46-ebcp

Antidepressive Agents, Second-Generation. Pharmacotherapy of panic disorder: proposed guidelines for the family physician (Roy-Byrne et al) 282-cr

Antidepressive Agents. Pharmacotherapy of panic disorder: proposed guidelines for the family physician (Roy-Byrne et al) 282-cr

The pharmacologic treatment of depression. ( $\mathrm{Ma}$ jeroni \& Hess) 127-cr

Anxiety. Use of the health history as a psychiatric screening tool (Post \& Miller) 452-oa

Asian Americans. Not just a fluke (Magill \& Roberts) 406-br 
423-c

Benefits of lipid-lowering therapy (Stein \& McBride)

Attending Physicians, Hospital. The emerging role of hospitalists - will family physicians continue to practice hospital medicine? (McConaghy) 324-refl

Bites and Stings. Dog bites: hidden danger of fulminant sepsis (Tisovec \& Kakaiya) 152-br; Man's best friend? (Blackman) 167-e

Blood Pressure Determination. Accuracy of blood pressure measurement in the family practice center (Kay) 252-oa

Board News. A conversation with the ABFP executive director (Avant) 420-bn

Pisacano Memorial Foundation - continuing the tradition (Cattoi) 490-bn

Book Reviews. Basic Cancer Medicine (Markman) [Driscoll] 83-bkrv

Breast Disease (Marchant) [Herold] 251-bkrv

Cbronic Musculoskeletal Injuries in the Workplace (Ranney) [Carek] 173-bkrv

The Computer-Based Patient Record: An Essential Technology for Health Care, Revised edition. (Dick et al) [Norris] 333-bkrv

Conn's Current Therapy 1998. Latest Approved Methods of Treatment for the Practicing Pbysician (Rakel) [Bergman] 498-bkrv

Current Diagnosis 9 (Conn et al) [Farley] 83-bkrv

Disorders of Mental Status: Dementia, Encephalopatby, Coma, Syncope (Misulis) [deGruy] 499-bkrv bkrv

Essentials of Musculoskeletal Care (Snider) [Eiff] 251-

Fracture Management for Primary Care (Eiff et al) [Fields] 334-bkrv

Fundamentals of Clinical Practice: $A$ Textbook on the Patient, Doctor and Society (Mengel \& Holleman) [Cate] 84bkrv

Griffitb's 5 Minute Clinical Consult 1998. (Dambro) [Frey] 333-bkrv

Instructions for Patients. Fifth edition. (Spanish language.) (Griffith et al) [Ruiz \& Gomez] 425-bkrv

International Occupational and Environmental Medicine

(Herzstein et al) [Lessenger] 335-bkrv

Manual of Skin Surgery - A Practical Guide to Dermatologic Procedures (Leffell \& Brown) [Fields] 84-bkrv A Measure of My Days: The Fournal of a Country Doctor (Loxterkamp) [Phillips] 84-bkrv

Nelson - Essentials of Pediatrics. Third edition. (Behrman \& Kliegman) [Davis] 498-bkrv

The 1998 CLC Integrative Medicine: A Balanced Account of the Data. (Wirth \& Farkas) [Kahn] 334-bkrv bkrv

Ophthalmology for Primary Care (Wu) [Fields] 174-

Patients Are a Virtue: Practicing Medicine in the Pennsylvania Amish Country (Wentz) [Hunt] 85-bkrv

Pediatrics. An Approacb to Independent Learning (Daeschner Jr \& Richardson) [Madlon-Kay] 173-bkrv

Primary Care Dermatology. (Arndt et al) [Driscoll] 500-bkrv

Primary Care Psychiatry (Knesper et al) [Zervanos] 173-bkrv
Saunders Manual of Pediatric Practice. (Finberg) [Acosta] 425-bkrv

Surviving the Fall: The Personal Fourney of an AIDS Doctor (Selwyn) [Carmichael] 499-bkrv

12-Lead ECG's - A Pocket Brain for Easy Interpretation (Grauer) [Eggertsen] 500-bkrv

BOOP - see Bronchiolitis Obliterans Organizing Pneumonia.

Brain Abscess. Tuberculous cerebellar abscess (Oshinowo et al) 459-mp

Breath Tests. Breath test and serologic testing for Helicobacter pylori (Ebell) 170-c; (Felz et al) 170-c

Brief Reports. Acute renal failure in an alcoholic patient taking therapeutic doses of acetaminophen (Maze \& Lee) 410-br

Acute toxicity from home-brewed Gamma hydroxybutyrate (Hodges \& Everett) 154-br

Acute-onset persistent olfactory deficit resulting from multiple overexposures to ammonia vapor at work (Prudhomme et al) 66-br

Erythema multiforme: case report and discussion (Kroonen) 63-br

Guillain-Barré syndrome and SIADH in a patient with chronic lymphocytic leukemia (Gutknecht) 237-br

Hematuria associated with sickle cell trait during pregnancy (Videlefsky \& Blount) 321-br

Metformin-associated lactic acidosis (Hulisz et al) 233-br

Not just a fluke (Magill \& Roberts) 406-br

Olfactory neuroblastoma as acute postpartum depression (Dascalu et al) 414-br

Recognition and management of oral health problems in older adults by physicians: a pilot study Jones et al) 474-br

Tetanus immunization status and predictors of immunity in older family practice outpatients (Costa \& Gerson) 315-br

Thrombolysis in acute ischemic stroke (Luisi \& Hume) 145-step

Transient hyperthyroidism in a patient taking dietary supplements containing kelp (Eliason) 478-br

Unintentional carbon monoxide poisoning from an unlikely source (Struttman et al) 481-br

An unusual cause of ureter rupture and acute abdomen (Fink) 318-br

Bronchiolitis Obliterans Organizing Pneumonia. Bronchiolitis obliterans organizing pneumonia mimicking community-acquired pneumonia (Ward \& Rog) 41-mp

Capnocytophaga. Dog bites: hidden danger of fulminant sepsis (Tisovec \& Kakaiya) 152-br; Man's best friend? (Blackman) 167-e

Carbidopa. Management of Parkinson disease (Mandanas) 171-c; (Manyam) 171-c

Carbon Monoxide Poisoning. Unintentional carbon monoxide poisoning from an unlikely source (Struttman et al) 481-br

Carboxyhemoglobin. Unintentional carbon monoxide poisoning from an unlikely source (Struttman et al) 481-br 
Cardiovascular Disease. Homocysteine and cardiovascular disease (Abby et al) 391-step

Cardiovascular Diseases/drug therapy. Evidencebased medicine in primary care: an overview (Geyman) 46-ebcp

Cerebral Ischemia. Thrombolysis in acute ischemic stroke (Hicks) 422-c; (Hume \& Luisi) 422-c

Thrombolysis in acute ischemic stroke (Luisi \& Hume) 145-step

Cerebrovascular Disorders. Thrombolysis in acute ischemic stroke (Luisi \& Hume) 145-step; Thrombolysis in acute ischemic stroke (Hicks) 422-c; (Luisi \& Hume) 422-c

Cervical Cancer. Determinants of cancer screening frequency: the example of screening for cervical cancer (Frame \& Frame) 87-oa

Chickenpox Vaccination. Varicella susceptibility and vaccination strategies in young adults (Jerant et al) 296-ebcp

Chiropractic. The use of alternative health care by a family practice population (Drivdahl \& Miser) 193-oa; Alternative medicine and the family physician (Jonas) 244-e

Cholinergic Antagonists. Management of Parkinson disease (Mandanas) 171-c; (Manyam) 171-c

Clinical Competence. On-site colposcopy services (Rodney) 80-c; (Scarpinato) 81-c

Clinical Practice Variations - see Physician Practice Patterns.

Clinical Reviews. Advances in office anesthesia (Whealton) 200-cr

Frostbite: review and current concepts (Reamy) 34-cr Medical consultation for the elderly patient with hip fracture (Ackermann) 366-oa

The pharmacologic treatment of depression. (Majeroni \& Hess) 127-cr

Pharmacotherapy of panic disorder: proposed guidelines for the family physician (Roy-Byrne et al) 282-cr

Clonorchiasis. Not just a fluke (Magill \& Roberts) 406-br

Cocaine. Advances in office anesthesia (Whealton) 200-cr

Colchicine/therapeutic use. Use of colchicine to treat severe constipation in developmentally disabled patients (Frame et al) 341-oa

Colposcopy. Determinants of cancer screening frequency: the example of screening for cervical cancer (Frame \& Frame) 87-oa

Efforts to improve the follow-up of patients with abnormal Papanicolaou test results (Block \& Branham) 1oa; Process instead of prayer: moving toward active management of patient care (Frame) 77-e

On-site colposcopy services (Rodney) 80-c; (Scarpinato) $81-c$

Community Health Planning. Putting populationbased care into practice: real option or rhetoric? (Taplin et al) 116-oa

Community Health Services. Tools for community-oriented primary care: a process for linking practice and community data (Mettee et al) 28-oa
Complementary Medicine - see Alternative Medicine.

Constipation. Use of colchicine to treat severe constipation in developmentally disabled patients (Frame et al) 341-oa

Contraceptives, Oral. Hormonal therapy in the management of premenstrual syndrome (Tiemstra \& Patel) 378-mp

Coronary Disease. Measurement and management of hyperlipidemia for the primary prevention of coronary heart disease (Froom et al) 12-oa; Benefits of cholesterol screening and therapy for primary prevention of cardiovascular disease: a new paradigm (Stein \& McBride) 72-e; Benefits of lipid-lowering therapy (Stein \& McBride) 423-c

Screening for dyslipidemia (Froom et al) $250-c$

Cost-Benefit Analysis. On-site colposcopy services (Rodney) 80-c; (Scarpinato) 81-c

Costs And Cost Analysis. Varicella susceptibility and vaccination strategies in young adults (Jerant et al) 296-ebcp

Credentialing. A conversation with the ABFP executive director (Avant) 420-bn

Current Report - HIV. Individualized strategies in the era of combination antiretroviral therapy (Goldschmidt et al) 158-crHIV

Death. Put to sleep (Magill) 165-refl

Death With Dignity. A gastrostomy in every stomach? (Brummel-Smith) 242-e

Decision Making. Evidence-based medicine in primary care: an overview (Geyman) 46-ebcp

Decision-Support Techniques. Evidence-based medicine in primary care: an overview (Geyman) 46ebcp

Delivery of Health Care. Inpatient practice and hospitalists (Ruane) 81-c

Dementia, Senile. Evaluating driving performance of outpatients with Alzheimer disease (Cox et al) 264oa

Depression, Postpartum. Olfactory neuroblastoma as acute postpartum depression (Dascalu et al) 414-br

Depression. The pharmacologic treatment of depression. (Majeroni \& Hess) 127-cr

Use of the health history as a psychiatric screening tool (Post \& Miller) 452-oa

Diabetes Mellitus. Barriers to recognition of erectile dysfunction among diabetic Mexican-American men (Zweifler et al) 259-oa; Sexual dysfunction in patients with diabetes (Herter) 327-e

Diabetes Mellitus, Non-Insulin Dependent. Metformin-associated lactic acidosis (Hulisz et al) 233-br

Diagnosis, Oral. Oral health care issues in HIV disease: developing a core curriculum for primary care physicians (Sifri et al) 434-oa.

Recognition and management of oral health problems in older adults by physicians: a pilot study Jones et al) 474-br

Dietary Supplementation - sce Food, Fortified. Dimethylguanylguanidine - see Metformin. 
Doctor-Patient Relations - see Physician-Patient Relations.

Dopamine Agonists. Management of Parkinson disease (Mandanas) 171-c; (Manyam) 171-c

Drug Abuse, Sports. Acute toxicity from homebrewed Gamma hydroxybutyrate (Hodges \& Everett) 154-br

Drug Combinations. Approach to polypharmacy (Solomon) 331-c; (Lee) 331-c

Drug Interactions. The pharmacologic treatment of depression. (Majeroni \& Hess) 127-cr

Drug Therapy, Combination. Approach to polypharmacy (Solomon) 331-c; (Lee) 331-c

Individualized strategies in the era of combination antiretroviral therapy (Goldschmidt et al) 158-crHIV

Polypharmacy: a case report and new protocol for management (Lee) 140-mp; Polypharmacy: old bad habits (Meador) 166-e

Drug Therapy. Evidence-based medicine in primary care: an overview (Geyman) 46-ebcp

Editorials. Alternative medicine and the family physician (Jonas) 144-e

Benefits of cholesterol screening and therapy for primary prevention of cardiovascular disease: a new paradigm (Stein \& McBride) 72-e

Family physicians' cholesterol testing and treatment (Crouch) 247-e 242-e

A gastrostomy in every stomach? (Brummel-Smith)

Man's best friend? (Blackman) 167-e

Polypharmacy: old bad habits (Meador) 166-e

Process instead of prayer: moving toward active management of patient care (Frame) $77-e$

Sexual dysfunction in patients with diabetes (Herter) 327-e

Will virtual reality simulators end the credentialing arms race in gastrointestinal endoscopy or the need for family physician faculty with endoscopic skills? (Rodney) 492-e

Education, Graduate. Virtual reality flexible sigmoidoscopy simulator training: impact on resident performance (Tuggy) 426-oa; Will virtual reality simulators end the credentialing arms race in gastrointestinal endoscopy or the need for family physician faculty with endoscopic skills? (Rodney) 492-e

Education, Medical, Graduate. A case for the development of family practice rural training tracks (Damos et al) 399-sp

Education, Medical. The 1-hour advising session: helping students choose family practice (Scherger) 70refl

Elderly - see Aged.

Enteral Nutrition. Gastrostomies in older patients: the 1990 National Hospital Discharge Survey (Grant) 187-oa; A gastrostomy in every stomach? (BrummelSmith) 242-e

Enthesopathy - see Rheumatic Diseases.

Environmental Exposure. Unintentional carbon monoxide poisoning from an unlikely source (Struttman et al) 481-br
Epidemiology. Putting population-based care into practice: real option or rhetoric? (Taplin et al) 116-oa

Epinephrine. Advances in office anesthesia (Whealton) 200-cr

Erectile Dysfunction - See Impotence.

Erythema Multiforme in infancy \& childhood. Erythema multiforme: case report and discussion (Kroonen) 63-br

Esthesioneuroblastoma. Olfactory neuroblastoma as acute postpartum depression (Dascalu et al) 414-br

Estrogens, Conjugated. Hormonal therapy in the management of premenstrual syndrome (Tiemstra \& Patel) 378-mp

Euthanasia, Passive. A gastrostomy in every stomach? (Brummel-Smith) 242-e

Evidence-Based Clinical Practice. Conservative versus surgical treatment of mallet finger: a pooled quantitative literature evaluation (Geyman et al) $382-$ ebcp

Dimensions of evidence (Berg) 216-ebcp

Evidence-based medicine in primary care: an overview (Geyman) 46-ebcp

Using outcomes to improve quality of research and quality of care (Deyo) 465-ebcp

Varicella susceptibility and vaccination strategies in young adults (Jerant et al) 296-ebcp

Evidence-Based Medicine. Dimensions of evidence (Berg) 216-ebcp

Evidence-based medicine in primary care: an overview (Geyman) 46-ebcp

Exercise. Clinical feasibility of a free-weight strengthtraining program for seniors (Brill et al) 445-oa

Family Practice. The 1-hour advising session: helping students choose family practice (Scherger) 70-refl

Border crossings: on the boundary of the physicianpatient relationship (Loxterkamp) 487-refl

Computerized prompts for cancer screening in a community health center (Yarnall et al) 96-oa

The doctor, the patient, and the home (White) 172-c

The emerging role of hospitalists - will family physicians continue to practice hospital medicine? (McConaghy) 324-refl

Health problems of refugees (Griswold et al) 171-c

Inpatient practice and hospitalists (Ruane) 81-c

Legacy of the secret war: medical needs in the UXOcontaminated areas in Laos (Morikawa) 485-w

The physician-patient relationship - perspectives after 40 years of family practice (Mgebroff) 417-refl

Training residents to care for handicapped patients (Kerle) 172-c

The use of alternative health care by a family practice population (Drivdahl \& Miser) 193-oa; Alternative medicine and the family physician (Jonas) 244-e

Family Practice World Perspective. Legacy of the secret war: medical needs in the UXO-contaminated areas in Laos (Morikawa) 485-w

Federal Health Insurance Plans, United States see National Health Insurance, United States.

Fibrosis. An unusual cause of ureter rupture and acute abdomen (Fink) 318-br 
Finger Injuries. Conservative versus surgical treatment of mallet finger: a pooled quantitative literature evaluation (Geyman et al) 382-ebcp

Flukes - see Trematoda Infections/diagnosis.

Folic Acid. Homocysteine and cardiovascular disease (Abby et al) 391-step

Food, Fortified. Transient hyperthyroidism in a patient taking dietary supplements containing kelp (Eliason) 478-br

Frostbite. Frostbite: review and current concepts (Reamy) 34-cr

gamma-Hydroxybutyrate - see Sodium Oxybate.

Gastrostomy. Gastrostomies in older patients: the 1990 National Hospital Discharge Survey (Grant) 187oa; A gastrostomy in every stomach? (Brummel-Smith) 242-e

Geriatric Assessment. Medical consultation for the elderly patient with hip fracture (Ackermann) 366cr

Geriatrics. Clinical feasibility of a free-weight strength-training program for seniors (Brill et al) 445oa

Recognition and management of oral health problems in older adults by physicians: a pilot study Jones et al) 474-br

Tetanus immunization status and predictors of immunity in older family practice outpatients (Costa \& Gerson) 315-br

Glucocorticoids, Synthetic. The use of corticosteroids in the management of herpes zoster (MacFarlane et al) 224-step

Gram-Negative Bacterial Infections. Dog bites: hidden danger of fulminant sepsis (Tisovec \& Kakaiya) 152-br; Man's best friend? (Blackman) 167-e

Guillain-Barré Syndrome - see Polyradiculoneuritis. Guillain-Barré syndrome and SIADH in a patient with chronic lymphocytic leukemia (Gutknecht) 237-br

Hazardous Substances. Acute-onset persistent olfactory deficit resulting from multiple overexposures to ammonia vapor at work (Prudhomme et al) 66-br; Irritant-induced anosmia (Edwards) 497-c; (Blanc) 497-c

Health Care Reform. 2001: a health odyssey? (Rabinowitz) 229-hp

Health Care Seeking Behavior - see Patient Acceptance of Health Care.

Health Care Surveys. Cholesterol testing and management: a national comparison of family physicians, general internists, and cardiologists (Eaton et al) 180-oa; Family physicians' cholesterol testing and treatment (Crouch) 247-e

Health Planning. Putting Population-based care into practice: real option or rhetoric? (Taplin et al) 116-oa

Tools for community-oriented primary care: a process for linking practice and community data (Mettee et al) 28-oa

Health Planning Technical Assistance. Tools for community-oriented primary care: a process for linking practice and community data (Mettee et al) 28-oa

Health Policy. 2001: a health odyssey? (Rabinowitz) 229-hp

Health Promotion. Computerized prompts for cancer screening in a community health center (Yarnall et al) $96-\mathrm{oa}$

Determinants of cancer screening frequency: the example of screening for cervical cancer (Frame \& Frame) 87-oa

Health Risk Appraisal. Cholesterol testing and management: a national comparison of family physicians, general internists, and cardiologists (Eaton et al) 180-oa; Family physicians' cholesterol testing and treatment (Crouch) 247-e

Helicobacter pylori. Breath test and serologic testing for Helicobacter pylori (Ebell) 170-c;(Felz et al) 170-c

Hematuria/in pregnancy. Hematuria associated with sickle cell trait during pregnancy (Videlefsky \& Blount) 321-br

Herpes Zoster. The use of corticosteroids in the management of herpes zoster (MacFarlane et al) 224step

Hip Fractures. Medical consultation for the elderly patient with hip fracture (Ackermann) 366-cr

HIV Infection. Oral health care issues in HIV disease: developing a core curriculum for primary care physicians (Sifri et al) 434-oa.

HIV. Individualized strategies in the era of combination antiretroviral therapy (Goldschmidt et al) 158crHIV

HMG CoA Reductases - see Hydroxymethylglutaryl CoA Reductases.

Homeless Persons. Frostbite: review and current concepts (Reamy) 34-cr

Homocysteine. Homocysteine and cardiovascular disease (Abby et al) 391-step

Hospice Care. The doctor, the patient, and the home (White) 172-c

Hospitalization. Inpatient practice and hospitalists (Ruane) 81-c

Hydroxymethylglutaryl CoA Reductases. Evidence-based medicine in primary care: an overview (Geyman) 46-ebcp

Hypercholesterolemia. Cholesterol testing and management: a national comparison of family physicians, general internists, and cardiologists (Eaton et al) 180-oa; Family physicians' cholesterol testing and treatment (Crouch) 247-e

Hyperlipidemia. Benefits of lipid-lowering therapy (Stein \& McBride) 423-c

Measurement and management of hyperlipidemia for the primary prevention of coronary heart disease (Froom et al) 12-oa; Benefits of cholesterol screening and therapy for primary prevention of cardiovascular disease: a new paradigm (Stein \& McBride) 72-e; Screening for dyslipidemia (Froom et al) 250-c

Hyperthyroidism. Prevalence of iatrogenic hyperthyroidism in a community hospital (Watsky \& Koeniger) 175-oa

Transient hyperthyroidism in a patient taking dietary 
supplements containing kelp (Eliason) 478-br

Iatrogenic Disease. Prevalence of iatrogenic hyperthyroidism in a community hospital (Watsky \& Koeniger) 175-oa

Immunization/active/in old age. Tetanus immunization status and predictors of immunity in older family practice outpatients (Costa \& Gerson) 315-br

Impotence/drug therapy. Barriers to recognition of erectile dysfunction among diabetic Mexican-American men (Zweifler et al) 259-oa; Sexual dysfunction in patients with diabetes (Herter) 327-e

Oral medications in the management of erectile dysfunction (Lawless \& Cree) 307-step

Inappropriate ADH Syndrome. Guillain-Barré syndrome and SIADH in a patient with chronic lymphocytic leukemia (Gutknecht) 237-br

Indians, North American. A gathering of eagles (Saal) 240-refl

Inflammation. Osteitis pubis: a diagnosis for the family physician (Andrews \& Carek) 291-mp

International Health Problems - see World Health.

Internship and Residency. A case for the development of family practice rural training tracks (Damos et al) 399-sp

The 1-hour advising session: helping students choose family practice (Scherger) 70-refl

On-site colposcopy services (Rodney) 80-c; (Scarpinato) 81-c

Training residents to care for handicapped patients (Kerle) 172-c

Virtual reality flexible sigmoidoscopy simulator training: impact on resident performance (Tuggy) 426-oa; Will virtual reality simulators end the credentialing arms race in gastrointestinal endoscopy or the need for family physician faculty with endoscopic skills? (Rodney) 492-e

Iontophoresis. Advances in office anesthesia (Whealton) 200-cr

Kidney Failure, Acute. Acute renal failure in an alcoholic patient taking therapeutic doses of acetaminophen (Maze \& Lee) 410-br

Lactic Acidosis - see Acidosis, Lactic.

Leukemia, Lymphocytic, Chronic. Guillain-Barré syndrome and SIADH in a patient with chronic lymphocytic leukemia (Gutknecht) 237-br

Levodopa. Management of Parkinson disease (Mandanas) 171-c; (Manyam) 171-c

Levothyroxine. Prevalence of iatrogenic hyperthyroidism in a community hospital (Watsky \& Koeniger) 175-oa

Lidocaine. Advances in office anesthesia (Whealton) 200-cr

Mammography. Computerized prompts for cancer screening in a community health center (Yarnall et al) 96-oa

Massage. The use of alternative health care by a family practice population (Drivdahl \& Miser) 193-oa; Alternative medicine and the family physician (Jonas) 244-e

Medical History Taking. New-patient self-history questionnaires in primary care (Guthmann) 23-oa

Medical History Taking/diagnostic use. Use of the health history as a psychiatric screening tool (Post \& Miller) 452-oa

Medical Practice. Approaches to urinary incontinence in a rural population: a comparison of physician assistants, nurse practitioners, and family physicians Jones \& Bunner) 207-mp

Bronchiolitis obliterans organizing pneumonia mimicking community-acquired pneumonia (Ward \& Rog) 41-mp

Hormonal therapy in the management of premenstrual syndrome (Tiemstra \& Patel) 378-mp

Osteitis pubis: a diagnosis for the family physician (Andrews \& Carek) 291-mp

Polypharmacy: a case report and new protocol for management (Lee) 140-mp

Tuberculous cerebellar abscess (Oshinowo et al) 459$\mathrm{mp}$

Medicare. Influence of provider characteristics and insurance status on maternal serum alpha-fetoprotein screening Jenkins-Woelk et al) 357-oa

Medicine, Evidence-Based - see Evidence-Based Medicine.

Medicine, Herbal. The use of alternative health care by a family practice population (Drivdahl $\&$ Miser) 193-oa; Alternative medicine and the family physician (Jonas) 244-e

Mental Retardation. Training residents to care for handicapped patients (Kerle) 172-c

Use of colchicine to treat severe constipation in developmentally disabled patients (Frame et al) 341-oa

Meta-Analysis. Conservative versus surgical treatment of mallet finger: a pooled quantitative literature evaluation (Geyman et al) 382-ebcp

Metformin. Metformin-associated lactic acidosis (Hulisz et al) 233-br

Military Medicine. Frostbite: review and current concepts (Reamy) 34-cr

Varicella susceptibility and vaccination strategies in young adults (Jerant et al) 296-ebcp

Mouth Diseases. Recognition and management of oral health problems in older adults by physicians: a pilot study (Jones et al) 474-br

National Ambulatory Medical Care Survey - see Health Care Surveys.

National Health Insurance, United States. 2001: a health odyssey? (Rabinowitz) 229-hp

Native Americans. A gathering of eagles (Saal) 240refl

Neuralgia. The use of corticosteroids in the management of herpes zoster (MacFarlane et al) 224-step

Neuroblastoma, Olfactory - see Esthesioneuroblastoma.

NIDDM - see Diabetes Mellitus, Non-Insulin Dependent.

Nurse-Midwives. Influence of provider characteristics and insurance status on maternal serum alpha-fetoprotein screening (Jenkins-Woelk et al) 357-oa

Nursing Assessment. Approaches to urinary incon- 
tinence in a rural population: a comparison of physician assistants, nurse practitioners, and family physicians Jones \& Bunner) 207-mp

Nursing Audit. Accuracy of blood pressure measurement in the family practice center (Kay) 252-oa

Occupational Health. Unintentional carbon monoxide poisoning from an unlikely source (Struttman et al) 481-br

Olfaction. Acute-onset persistent olfactory deficit resulting from multiple overexposures to ammonia vapor at work (Prudhomme et al) 66-br; Irritant-induced anosmia (Edwards) 497-c; (Blanc) 497-c

Oral Health. Oral health care issues in HIV disease: developing a core curriculum for primary care physicians (Sifri et al) 434-oa.

Recognition and management of oral health problems in older adults by physicians: a pilot study Jones et al) 474-br

Osteitis. Osteitis pubis: a diagnosis for the family physician (Andrews \& Carek) 291-mp

Outcome Assessment (Health Care). Conservative versus surgical treatment of mallet finger: a pooled quantitative literature evaluation (Geyman et al) 382ebcp

Using outcomes to improve quality of research and quality of care (Deyo) 465-ebcp

Outcomes Research - see Outcome Assessment (Health Care).

Panic Disorder, Diagnosis. Pharmacotherapy of panic disorder: proposed guidelines for the family physician (Roy-Byrne et al) 282-cr

Panic Disorder/drug therapy. Pharmacotherapy of panic disorder: proposed guidelines for the family physician (Roy-Byrne et al) 282-cr

Papanicolaou Smears - see Vaginal Smears.

Parkinson Disease. Management of Parkinson disease (Mandanas) 171-c; (Manyam) 171-c

Patient Acceptance of Health Care. Influence of provider characteristics and insurance status on maternal serum alpha-fetoprotein screening Jenkins-Woelk et al) 357-oa

Understanding and caring for the distressed patient with multiple medically unexplained symptoms (Walker et al) 347-oa

Patient Care Team. Putting population-based care into practice: real option or rhetoric? (Taplin et al) 116-oa

Patient Compliance. On-site colposcopy services (Rodney) 80-c; (Scarpinato) 81-c

Patient Simulation. Approaches to urinary incontinence in a rural population: a comparison of physician assistants, nurse practitioners, and family physicians Jones \& Bunner) 207-mp

Pelvic Pain. Osteitis pubis: a diagnosis for the family physician (Andrews \& Carek) 291-mp

Peptic Ulcer. Breath test and serologic testing for Helicobacter pylori (Ebell) 170-c; (Felz et al) 170-c

Perceptual Motor Performance. Evaluating driving performance of outpatients with Alzheimer disease (Cox et al) 264-oa
Phentolamine. Oral medications in the management of erectile dysfunction (Lawless \& Cree) 307step

Physical Conditioning, Human - see Physical Fitness.

Physical Fitness. Clinical feasibility of a free-weight strength-training program for seniors (Brill et al) 445-oa

Physician Assistants. Approaches to urinary incontinence in a rural population: a comparison of physician assistants, nurse practitioners, and family physicians Jones \& Bunner) 207-mp

Physician Practice Patterns. Cholesterol testing and management: a national comparison of family physicians, general internists, and cardiologists (Eaton et al) 180-oa; Family physicians' cholesterol testing and treatment (Crouch) 247-e

Influence of provider characteristics and insurance status on maternal serum alpha-fetoprotein screening Jenkins-Woelk et al) 357-oa

On-site colposcopy services (Rodney) 80-c; (Scarpinato) 81-c

Primary care physicians and complementary-alternative medicine: training, attitudes, and practice patterns (Berman et al) 272-oa

Physician's Role. The doctor, the patient, and the home (White) 172-c

Physician-Patient Relations. Border crossings: on the boundary of the physician-patient relationship (Loxterkamp) 487-refl

The doctor, the patient, and the home (White) 172-c

The physician-patient relationship - perspectives after 40 years of family practice (Mgebroff) 417-refl

Understanding and caring for the distressed patient with multiple medically unexplained symptoms (Walker et al) 347-oa

Physicians, Family. Approaches to urinary incontinence in a rural population: a comparison of physician assistants, nurse practitioners, and family physicians (Jones \& Bunner) 207-mp

Cholesterol testing and management: a national comparison of family physicians, general internists, and cardiologists (Eaton et al) 180-oa; Family physicians' cholesterol testing and treatment (Crouch) 247-e

Primary care physicians and complementary-alternative medicine: training, attitudes, and practice patterns (Berman et al) 272-oa

Pisacano, Nicholas J. Pisacano Memorial Foundation - continuing the tradition (Cattoi) 490-bn

Pneumonia. Bronchiolitis obliterans organizing pneumonia mimicking community-acquired pneumonia (Ward \& Rog) 41-mp

Polymerase Chain Reaction. Tuberculous cerebellar abscess (Oshinowo et al) 459-mp

Polypharmacy. Polypharmacy: a case report and new protocol for management (Lee) 140-mp; Polypharmacy: old bad habits (Meador) 166-e; Approach to polypharmacy (Solomon) 331-c; (Lee) 331-c 
Polyradiculoneuritis. Guillain-Barré syndrome and SIADH in a patient with chronic lymphocytic leukemia (Gutknecht) 237-br

Population-Based Planning - see Community Health Planning.

Postpartum Depression - see Depression, Postpartum.

Practice Guidelines. Benefits of lipid-lowering therapy (Stein \& McBride) 423-c

Management of Parkinson disease (Mandanas) 171-c; (Manyam) 171-c

Measurement and management of hyperlipidemia for the primary prevention of coronary heart disease (Froom et al) 12-oa; Benefits of cholesterol screening and therapy for primary prevention of cardiovascular disease: a new paradigm (Stein \& McBride) 72 -e

Screening for dyslipidemia (Froom et al) 250-c

Practice Management, Medical. New-patient self-history questionnaires in primary care (Guthmann) 23-oa

On-site colposcopy services (Rodney) 80-c; (Scarpinato) $81-c$

Prednisone. The use of corticosteroids in the management of herpes zoster (MacFarlane et al) 224-step

Pregnancy. Smoking cessation intervention: the practices of maternity care providers (Helwig et al) 336-oa

Pregnancy Complications. Hematuria associated with sickle cell trait during pregnancy (Videlefsky \& Blount) 321-br

Pregnancy, High-Risk. Hematuria associated with sickle cell trait during pregnancy (Videlefsky \& Blount) 321-br

Premenstrual Syndrome. Hormonal therapy in the management of premenstrual syndrome (Tiemstra \& Patel) 378-mp

Prenatal Care. Influence of provider characteristics and insurance status on maternal serum alpha-fetoprotein screening (Jenkins-Woelk et al) 357-oa

Smoking cessation intervention: the practices of maternity care providers (Helwig et al) 336-oa

Prevention, Primary - see Primary Prevention.

Preventive Health Services. Computerized prompts for cancer screening in a community health center (Yarnall et al) 96-oa

Determinants of cancer screening frequency: the example of screening for cervical cancer (Frame \& Frame) 87-oa

Primary Health Care. Border crossings: on the boundary of the physician-patient relationship (Loxterkamp) 487-refl

Computerized prompts for cancer screening in a community health center (Yarnall et al) 96-oa

Health problems of refugees (Griswold et al) 171-c

Inpatient practice and hospitalists (Ruane) $81-c$

New-patient self-history questionnaires in primary care (Guthmann) 23-oa

Putting population-based care into practice: real option or rhetoric? (Taplin et al) 116-oa

The doctor, the patient, and the home (White) 172-c
Training residents to care for handicapped patients (Kerle) 172-c

Primary Prevention. Benefits of cholesterol screening and therapy for primary prevention of cardiovascular disease: a new paradigm (Stein \& McBride) 72-e

Benefits of lipid-lowering therapy (Stein \& McBride) 423-c

Computerized prompts for cancer screening in a community health center (Yarnall et al) 96-oa

Determinants of cancer screening frequency: the example of screening for cervical cancer (Frame $\&$ Frame) 87-oa

Measurement and management of hyperlipidemia for the primary prevention of coronary heart disease (Froom et al) 12-oa; Screening for dyslipidemia (Froom et al) 250-c

Progestational Hormones, Synthetic. Hormonal therapy in the management of premenstrual syndrome (Tiemstra \& Patel) 378-mp

Protease Inhibitors. Individualized strategies in the era of combination antiretroviral therapy (Goldschmidt et al) 158-crHIV

Psychomotor Performance/in old age. Evaluating driving performance of outpatients with Alzheimer disease (Cox et al) 264-oa

Psychosexual Dysfunctions. Oral medications in the management of erectile dysfunction (Lawless \& Cree) 307-step

Pubic Symphysis. Osteitis pubis: a diagnosis for the family physician (Andrews \& Carek) 291-mp

Quality Assurance, Health Care. Using outcomes to improve quality of research and quality of care (Deyo) 465-ebcp

Quality of Health Care. Using outcomes to improve quality of research and quality of care (Deyo) 465-ebcp

Questionnaires. New-patient self-history questionnaires in primary care (Guthmann) 23-oa

Reflections in Family Practice. Border crossings: on the boundary of the doctor-patient relationship (Loxterkamp) 487-refl

The emerging role of hospitalists - will family physicians continue to practice hospital medicine? (McConaghy) 324-refl

A gathering of eagles (Saal) 240-refl

The 1-hour advising session: helping students choose family practice (Scherger) 70-refl

The physician-patient relationship - perspectives after 40 years of family practice (Mgebroff) 417-refl

Put to sleep (Magill) 165-refl .

Refugees. Health problems of refugees (Griswold et al) 171-c

Reproducibility of Results. Accuracy of blood pressure measurement in the family practice center (Kay) 252-oa

Residency, Medical - see Internship and Residency.

Rheumatic Diseases. Osteitis pubis: a diagnosis for the family physician (Andrews \& Carek) 291-mp

Risk Factors. Benefits of lipid-lowering therapy (Stein \& McBride) 423-c 
Screening for dyslipidemia (Froom et al) 250-c

Rural Health Services. A case for the development of family practice rural training tracks (Damos et al) 399-sp

Computerized prompts for cancer screening in a community health center (Yarnall et al) 96-oa

Seaweed. Transient hyperthyroidism in a patient taking dietary supplements containing kelp (Eliason) 478-br

Selegiline. Management of Parkinson disease (Mandanas) 171-c; (Manyam) 171-c

Self-Care. Clinical feasibility of a free-weight strength-training program for seniors (Brill et al) 445-oa

Sepsis. Dog bites: hidden danger of fulminant sepsis (Tisovec \& Kakaiya) 152-br; Man's best friend? (Blackman) 167-e

Serotonin Uptake Inhibitors. Pharmacotherapy of panic disorder: proposed guidelines for the family physician (Roy-Byrne et al) 282-cr

Sex Counseling. Barriers to recognition of erectile dysfunction among diabetic Mexican-American men (Zweifler et al) 259-oa; Sexual dysfunction in patients with diabetes (Herter) 327-e

Oral medications in the management of erectile dysfunction (Lawless \& Cree) 307-step

Sex Disorders. Oral medications in the management of erectile dysfunction (Lawless \& Cree) 307step

SIADH - see Inappropriate ADH Syndrome.

Sickle Cell Trait/in pregnancy. Hematuria associated with sickle cell trait during pregnancy (Videlefsky \& Blount) 321-br

Sigmoidoscopy. Virtual reality flexible sigmoidoscopy simulator training: impact on resident performance (Tuggy) 426-oa; Will virtual reality simulators end the credentialing arms race in gastrointestinal endoscopy or the need for family physician faculty with endoscopic skills? (Rodney) 492-e

Sildenafil. Oral medications in the management of erectile dysfunction (Lawless \& Cree) 307-step

Smoking Cessation. Computerized prompts for cancer screening in a community health center (Yarnall et al) 96-oa

Smoking cessation intervention: the practices of maternity care providers (Helwig et al) 336-oa

Sodium Oxybate. Acute toxicity from home-brewed Gamma hydroxybutyrate (Hodges \& Everett) 154-br

Somatoform Disorders. Understanding and caring for the distressed patient with multiple medically unexplained symptoms (Walker et al) 347-oa

Special Communications. A case for the development of family practice rural training tracks (Damos et al) 399-sc

STEPped Care: An Evidence-Based Approach to Drug Therapy. Homocysteine and cardiovascular disease (Abby et al) 391-step

Oral medications in the management of erectile dysfunction (Lawless \& Cree) 307-step

Reducing cardiovascular morbidity and mortality with the statins (Force) 57-step
Thrombolysis in acute ischemic stroke (Luisi \& Hume) 145-step

The use of corticosteroids in the management of herpes zoster (MacFarlane et al) 224-step

Street People - see Homeless Persons.

Stroke - see Cerebrovascular Disorders.

Students, Medical. The 1-hour advising session: helping students choose family practice (Scherger) 70-refl

Suicide. Put to sleep (Magill) 165-refl

Tetanus Toxoid/in old age. Tetanus immunization status and predictors of immunity in older family practice outpatients (Costa \& Gerson) 315-br

Tetracaine. Advances in office anesthesia (Whealton) 200-cr

Thrombolytic Therapy. Thrombolysis in acute ischemic stroke (Luisi \& Hume) 145-step; Thrombolysis in acute ischemic stroke (Hicks) 422-c; (Luisi \& Hume) $422-c$

Thymoleptics - see Antidepressive Agents.

Toxic Substances, Environmental - see Hazardous Substances.

Trematoda Infections/diagnosis. Not just a fluke (Magill \& Roberts) 406-br

Tuberculosis. Tuberculous cerebellar abscess (Oshinowo et al) 459-mp

Ureter/pathology. An unusual cause of ureter rupture and acute abdomen (Fink) 318-br

Ureteral Obstruction. An unusual cause of ureter rupture and acute abdomen (Fink) 318-br

Urinary Diversion. An unusual cause of ureter rupture and acute abdomen (Fink) 318-br

Urinary Incontinence. Approaches to urinary incontinence in a rural population: a comparison of physician assistants, nurse practitioners, and family physicians (Jones \& Bunner) 207-mp

User-Computer Interface. Virtual reality flexible sigmoidoscopy simulator training: impact on resident performance (Tuggy) 426-oa; Will virtual reality simulators end the credentialing arms race in gastrointestinal endoscopy or the need for family physician faculty with endoscopic skills? (Rodney) 492-e

Vaccination/in old age. Tetanus immunization status and predictors of immunity in older family practice outpatients (Costa \& Gerson) 315-br

Vaginal Smears. Computerized prompts for cancer screening in a community health center (Yarnall et al) 96-oa

Determinants of cancer screening frequency: the example of screening for cervical cancer (Frame \& Frame) 87-oa

Efforts to improve the follow-up of patients with abnormal Papanicolaou test results (Block \& Branham) 1oa; Process instead of prayer: moving toward active management of patient care (Frame) 77-e

Varicella Vaccination. Varicella susceptibility and vaccination strategies in young adults (Jerant et al) 296ebcp

Virtual Systems. Virtual reality flexible sigmoidoscopy simulator training: impact on resident perfor- 
mance (Tuggy) 426-oa; Will virtual reality simulators end the credentialing arms race in gastrointestinal endoscopy or the need for family physician faculty with endoscopic skills? (Rodney) 492-e

Visual Motor Coordination. Evaluating driving performance of outpatients with Alzheimer disease (Cox et al) 264-oa

World Health. Health problems of refugees (Griswold et al) 171-c

Legacy of the secret war: medical needs in the UXOcontaminated areas in Laos (Morikawa) 485-w

Wounds, Penetrating. Legacy of the secret war: medical needs in the UXO-contaminated areas in Laos (Morikawa) 485-w

Yohimbine. Oral medications in the management of erectile dysfunction (Lawless \& Cree) 307-step
STATEMENT OF OWNERSHIP, MANAGEMENT, AND CIRCULATION (Required by 39 U.S.C. 3685 ).

1. Publication title: The Journal of the American Board of Family Practice

2. Publication number: 0893-8652.

3. Date of filing: September 28, 1998.

4. Issue frequency: Bimonthly.

5. Number of issues published annually: 6 .

6. Annual subscription price: Domestic: $\$ 60 / \$ 35 / \$ 20$. International: $\$ 65 / \$ 45 / \$ 45$.

7. Complete mailing address of known office of publication: Journal of the American Board of Family Practice, 2228 Young Dr., Lexington, KY 40505-4294.

8. Complete mailing address of headquarters or general business offices of publisher: 241 Forsgate Dr., Jamesburg, NJ 08831.

9. Full names and complete mailing addresses of publisher: Medical World Communications, 241 Forsgate Dr., Jamesburg. NJ 08831; Editor: John P. Geyman. MD, PO Box 354696, University of Washington, Seattle, WA 98195; Managing Editor: Paul R. Young. MD, 2228 Young Dr., Lexington, KY 40505-4294.

10. Owner: American Boand of Family Practice, Inc., 2228 Young Dr., Lexington, KY 40505-4294.

11. Known bondholders, mortgagees, and other security holders owning or holding $1 \%$ or more of total amounts of bonds, mortgages, or other securities: None.

12. Tax status: The purpose, function, and nonprofit status of this organization and the exempt status for federal income tax purposes has not changed during preceding 12 months.

13. Publication name: The Journal of the American Board of Family Practice.

14. Issue date for circulation data below: July/August 1998.

15. Extent and nature of circulation:

a. Average number of copies of each issue during preceding 12 months: 60,897 (net press run). Actual number of copies of single lisue published nearest to filing date: 60,830 (net press run).

b. Paid circulation: (1) sales through dealers and carriers, street vendors, and counter sales: None.

(2) Paid or requested mail subscriptions: 53,234 (average); 53,075 (actual single issue).

c. Total paid and/or requested circulation: 53,234 (average); 53,075 (actual single issue).

d. Free distribution by mail (Samples, complimentary, and other free copies: 7,195 (average); 7,293 (actual single issue).

e. Free distribution outside the mail: None

f. Total free distribution: 7,195 (average); 7,293 (actual single issue).

g. Total distribution: 60,429 (average); 60,368 (actual single issue).

h. Copies not distributed: 468 (average); 462 (actual single issue).

1. Total: 468 (average); 462 (actual single issue).

j. Percent paid and/or requested circulation: $88 \%$ (average); $88 \%$ (actual single copy).

I certify that all information furnished on this form is correct and complete.

(signed) Paul R. Young, MD, Managing Editor 